\title{
Focus the Mining Beacon: Lessons and Challenges from the World of E-Commerce
}

\author{
Ron Kohavi \\ Microsoft Corporation, USA \\ ronnyk@cs.stanford.edu \\ http://www.kohavi.com
}

Electronic Commerce is now entering its second decade, with Amazon.com and eBay now in existence for ten years. With massive amounts of data, an actionable domain, and measurable ROI, multiple companies use data mining and knowledge discovery to understand their customers and improve interactions. We present important lessons and challenges using e-commerce examples across two dimensions: (i) business-level to technical, and (ii) the mining lifecycle from data collection, data warehouse construction, to discovery and deployment. Many of the lessons and challenges are applicable to domains outside e-commerce. 